\begin{tabular}{|c|l|}
\hline Title & $\begin{array}{l}\text { Growth kinetics and theoretical modeling of selective molecular beam epitaxy for growth of GaA s nanowires on } \\
\text { nonplanar (001) and (111)B substrates }\end{array}$ \\
\hline Author(s) & Sato, Taketomo; Tamai, Isao; Hasegawa, Hideki \\
\hline Citation & $\begin{array}{l}\text { Journal of Vacuum Science \& Technology B: Microelectronics and Nanometer Structures, 23(4), 1706-1713 } \\
\text { https:/doi.org/L0.1116/1.1949222 }\end{array}$ \\
\hline Issue Date & 2005-07 \\
\hline Doc URL & http://hdl.handle.net/2115/8381 \\
\hline Rights & ○ 2005 A merican V acuum Society \\
\hline Type & article \\
\hline File Information & TSA TO7.PDF \\
\hline
\end{tabular}

Instructions for use 


\title{
Growth kinetics and theoretical modeling of selective molecular beam epitaxy for growth of GaAs nanowires on nonplanar (001) and (111)B substrates
}

\author{
Taketomo Sato, ${ }^{\text {a) }}$ Isao Tamai, and Hideki Hasegawa \\ Research Center for Integrated Quantum Electronics (RCIQE) and Graduate School Information Science \\ and Technology, Hokkaido University, North 13, West 8, Kita-ku, Sapporo 060-8628, Japan
}

(Received 23 January 2005; accepted 28 April 2005; published 25 July 2005)

\begin{abstract}
The growth kinetics involved in the selective molecular beam epitaxy growth of GaAs quantum wires (QWRs) on mesa-patterned substrates is investigated in detail experimentally, and an attempt is made to model the growth theoretically, using a phenomenological continuum model. Experimentally, $\langle-110\rangle$-oriented QWRs were grown on (001) and (113)A substrates, and $\langle-1-12\rangle$-oriented QWRs were grown on (111)B substrates. From a detailed investigation of the growth profiles, it was found that the lateral wire width is determined by facet boundaries (FBs) within AlGaAs layers separating growth regions on top facets from those on side facets of mesa structures. Evolution of FBs during growth was complicated. For computer simulation, measured growth rates of various facets were fitted into a theoretical formula to determine the dependence of a lifetime of adatoms on the slope angle of the growing surface. The continuum model using the slope angle dependent lifetime reproduced the details of the experimentally observed growth profiles very well for growth on (001), (113)A, and (111)B substrates, including the complex evolution of facet boundaries (C) 2005 American Vacuum Society. [DOI: 10.1116/1.1949222]
\end{abstract}

\section{INTRODUCTION}

Selective growth of III-V semiconductors on prepatterned substrates either by molecular beam epitaxy (MBE) or by metal organic vapor phase epitaxy is one of the most promising techniques for formation of position- and sizecontrolled arrays of quantum wires (QWRs) and quantum dots. ${ }^{1-4}$ Recently, we have reported successful selective MBE growth of InP-based ${ }^{5-7}$ and GaAs-based ${ }^{8-10}$ QWRs. They included $\langle-110\rangle$-oriented QWRs on (001) substrates and $\langle-1-12\rangle$-oriented wires on (111)B substrates. The minimum wire width was below $10 \mathrm{~nm}$ (Ref. 6) and the maximum amount of blueshift of the photoluminescence (PL) peak energy due to quantum confinement was $440 \mathrm{meV}$, including a significant contribution from lateral confinement. Typical full width at half maximum values of the PL emission peaks from QWRs have been very narrow being below $30 \mathrm{meV}$ for QWR arrays where the photoexited areas typically included more than 100 QWRs with wire lengths over $100 \mu \mathrm{m}$, indicating that the cross sections of wires is highly uniform along their main axis. Direct observation by spatially resolved cathodoluminescence images also indicated uniformity of QWRs. ${ }^{5,9}$ Thus, these promising results indicate that such QWRs may become building blocks for future high density quantum nanoelectronics and nanophotonics.

For such purposes, precise control of wire cross section and feature sizes in nanometer scale is obviously very important. However, growth on nonplanar substrates usually involves various high-index facets simultaneously and this

\footnotetext{
a) Author to whom all correspondence should be addressed; electronic mail: taketomo@rciqe.hokudai.ac.jp
}

complicates growth kinetics. ${ }^{11}$ Thus, a quantitative modeling of the growth based on proper understanding of the underlying growth mechanism is a key issue.

Up to now, a large number of efforts on numerical modeling of the crystal growth on nonplanar substrates have been reported, using atomistic models ${ }^{12,13}$ and continuum models. ${ }^{14-19}$ In particular, use of diffusion equations under the continuum approximation with phenomenological macroscopic parameters such as diffusion constants, migration length, and incorporation rates has shown a considerable success, qualitatively reproducing on computer experimentally observed evolution of complex growth profiles of micronmeter sized structures. ${ }^{14,15}$ However, since the previous works emphasized mathematical aspects of modeling, comparison with experiments were rather poor and incomplete, and it is not clear how accurately a modeling based on the continuum approach can reproduce the experimentally observed evolution of cross-sectional features of nanometersized quantum structures. Especially, to establish a modeling method widely applicable to growth on any kind of crystalline facets, a systematic investigation of the growth behavior is necessary both experimentally and theoretically.

The purpose of this article is to experimentally investigate and theoretically model the evolution of cross-sectional features of GaAs nanowires during their growth by selective MBE on prepatterned nonplanar (001) and (111)B GaAs substrates. For this purpose, detailed basic growth experiments were carried out on mesa-patterned substrates of (001), (113)A, and (111)B orientations. Then, a phenomenological modeling based on the continuum approximation was attempted. Particular attention was paid on the dependence of the adatom lifetime on the slope angle of the growing surface. Computer simulation based on the resultant lifetime 


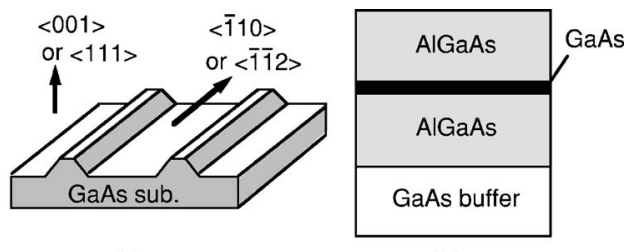

(a)

(b)

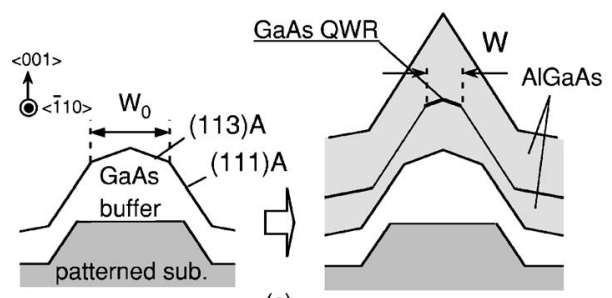

(c)

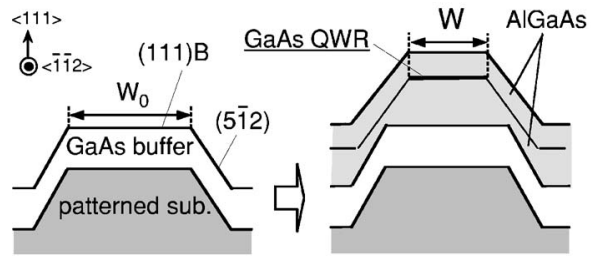

(d)

FIG. 1. Selective growth sequence used in this study. (a) Preparation of patterned substrate, (b) material supply, (c) growth of the $\langle-110\rangle$-oriented GaAs wire, and (d) growth of the $\langle-1-12\rangle$-oriented GaAs wire.

behavior successfully reproduced the experimentally observed growth features, indicating that the wire cross sections are kinetically controlled by growth conditions.

\section{EXPERIMENTAL STUDY OF SELECTIVE GROWTH}

\section{A. Growth method and conditions}

The sequence for selective MBE growth of GaAs QWRs used in this study is schematically shown in Figs. 1(a)-1(d). As a template for selective MBE growth, arrays of $\langle-110\rangle$ or $\langle-1-12\rangle$-oriented mesa stripes shown in Fig. 1(a) are formed on semi-insulating (001) or (111)B GaAs substrates. Patterns are prepared by the standard electron beam lithography and wet chemical etching.

Before loading into the MBE chamber, the patterned substrate was cleaned by acetone and ethanol with ultrasonic agitation, and then a light chemical etching was subsequently applied in the atmosphere, using a Semicoclean (Furuuchi Chemical Co., Ltd., Tokyo, Japan) solution. After loading into the MBE chamber, thermal cleaning was applied just prior to growth at a substrate temperature of $640{ }^{\circ} \mathrm{C}$ and under an arsenic pressure of $6 \times 10^{-4} \mathrm{~Pa}$, monitoring the reflective high energy electron diffraction patterns.

A typical material supply sequence is shown in Fig. 1(b). First, a GaAs buffer layer is grown on a patterned substrate in order to prepare a growth template for subsequent selective growth. In our previous study, ${ }^{8}$ buffer growth on the $\langle-110\rangle$-oriented mesa stripes on (001) substrates has led to a GaAs ridge structure defined by top (113)A and side (111)A facets, as shown in Fig. 1(c). Then, an $\mathrm{Al}_{0.3} \mathrm{Ga}_{0.7} \mathrm{As} / \mathrm{GaAs} /$ $\mathrm{Al}_{0.3} \mathrm{Ga}_{0.7} \mathrm{As}$ sandwich layer is grown on the buffer template, and this leads to the formation of embedded GaAs QWRs on the top (113)A facets of an AlGaAs ridge structure with a reduced lateral wire width, as shown on the right in Fig. 1(c). As for buffer growth on $\langle-1-12\rangle$-oriented mesa stripes on (111)B substrates, ${ }^{20}$ it has led to a GaAs mesa structure defined by top (111)B and side (5-12) facets, as shown in Fig. $1(\mathrm{~d})$. Then, growth of an $\mathrm{Al}_{0.3} \mathrm{Ga}_{0.7} \mathrm{As} / \mathrm{GaAs} / \mathrm{Al}_{0.3} \mathrm{Ga}_{0.7} \mathrm{As}$ sandwich layer on this buffer template leads to formation of embedded GaAs QWRs on the top (111)B facets of an AlGaAs mesa structure with a reduced lateral wire width, as shown on the right in Fig. 1(d).

In the present study, growth of wires was repeated on the same patterned substrate in order to investigate evolution of their complex cross-sectional features during growth. Large mesa sizes were intentionally used so that a detailed crosssectional scanning electron microscopy (SEM) study could be made, using a Hitachi S-4100 microscope. In addition to the (001) and (111)B substrates, growth on patterns formed on the specially purchased (113)A substrate was carried out to clarify the growth behavior.

As the standard growth conditions, growth temperature $T_{\text {sub }}$ was set to be $640{ }^{\circ} \mathrm{C}$ for (001) and (113)A substrates and $700{ }^{\circ} \mathrm{C}$ for (111)B substrates. The growth rates of GaAs and AlGaAs layers were kept to be 700 and $1000 \mathrm{~nm} / \mathrm{h}$, respectively, in terms of the values for growth on a planar reference substrate which was placed next to the patterned substrate. The V/III ratio was set to be 30 for (001) and (113)A substrates and 10 for (111)B substrates.

\section{B. Wire cross-sections and facet boundaries}

Growth experiments on (001) and (111)B-oriented patterned substrates confirmed successful selective growth of $\langle-110\rangle$ - and $\langle-1-12\rangle$-oriented quantum wire arrays whose wire cross sections are shown in Figs. 1(c) and 1(d), respectively. To see how the wire width and position are determined, experiments of repeated wire growth on the same patterned substrate were carried out. The resultant crosssectional SEM images of the samples grown on a (001) patterned substrate and on a (111)B patterned substrate are shown in Figs. 2(a) and 2(b), respectively. In both cases, GaAs wires were selectively formed on the top facets of $\mathrm{AlGaAs}$ ridge or mesa structures with reduced sizes.

Furthermore, repeated growth experiments revealed the presence of boundaries separating the region grown on the top facets and that grown on the side facets within the AlGaAs barrier layer as indicated by dashed lines in Figs. 2(a) and 2(b). These lines are called here as the facet boundaries (FBs). As for growth on the (001) substrate, these boundaries are planes and the lateral wire width of QWRs, $W$, is determined by two facet boundary planes (FBPs) which form a constant angle $\theta_{b}$ with respect to the flat (001) plane. It has been found in previous experiments ${ }^{21}$ that these boundary planes do not correspond to any particular crystalline facets that are activated during the growth. In fact, they were found to depend strongly on the growth temperature $T_{\text {sub }}$. 


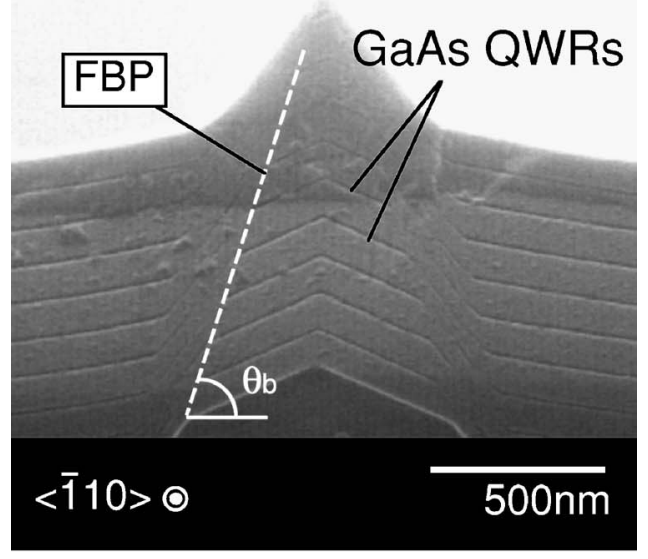

(a)

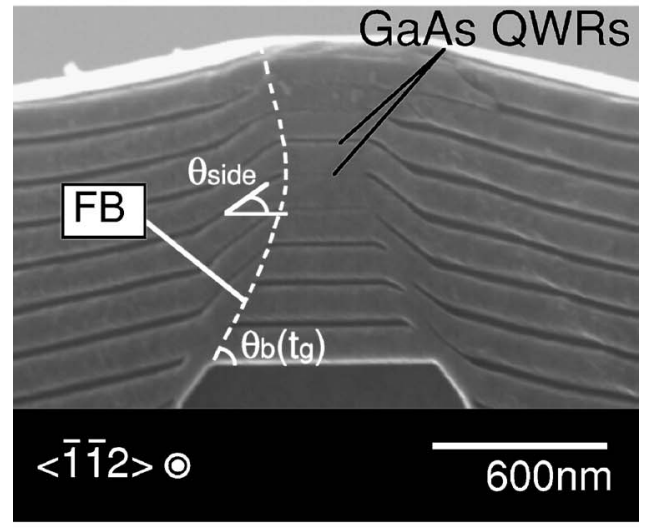

(b)

FIG. 2. Cross-sectional SEM images of samples after repeated growth of QWRs.: (a) $\langle-110\rangle$-oriented wires on the (001) substrate and (b) $\langle-1-12\rangle$ oriented wires on the (111)B substrate. The dashed lines indicate the facet boundaries.

On the other hand, the facet boundary is not planar for the growth on the (111)B substrate, as seen in Fig. 2(b). It is clear that the angle of side facets of the mesa with respect to the top facet $\theta_{\text {side }}$ decreases with the increase of the growth time $t_{g}$ leading to a time-dependent FB angle $\theta_{b}\left(t_{g}\right)$ as shown in Fig. 2(b) by the dashed curve. This phenomenon became more pronounced when the vertical height of mesa was reduced. It is obvious that these curved facet boundaries play important roles in determining the width and height of the wire for the growth on the (111)B substrate.

\section{Growth on one-sided mesa steps}

As shown in the previous section, evolution of the crosssectional structure of the QWR is very complicated. In order to get further information on growth kinetics including evolution of facet boundaries, we chose a simpler and more basic structure, i.e., a one-sided mesa step. Step structures were prepared not only on the (001) and (111)B substrates but also on the (113)A substrate, and growth experiments were carried out on these three structures. A $\langle-110\rangle$-oriented mesa step formed on a (113)A substrate has (111)A side facets similar to the case of mesa formed on a (001) substrate.

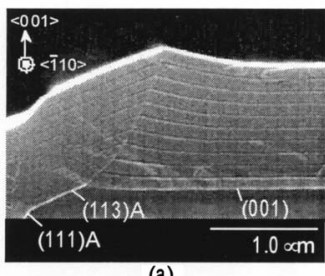

(a)

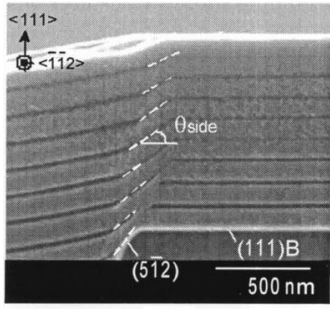

(c)

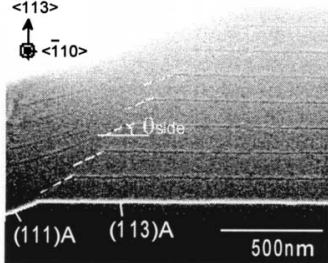

(b)

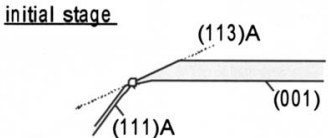

later

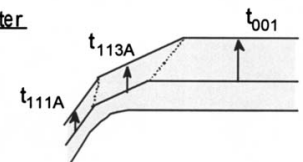

(d)
FIG. 3. Cross-sectional SEM images of samples after repeated growth of AlGaAs $(100 \mathrm{~nm}) / \mathrm{GaAs}(10 \mathrm{~nm})$ on basic one-sided mesa: (a) $\langle-110\rangle$ oriented mesa on a (001) substrate; (b) $\langle-110\rangle$-oriented mesa on (113)A substrate, and (c) $\langle-1-12\rangle$-oriented mesa on a (111)B substrate. (d) A likely mechanism for evolution of the (113)A facet for growth on (001) substrate.

Therefore, such a plane is useful for the investigation on the basic growth properties of the present $\langle-110\rangle$-oriented wire formed on the ridge structure having top (113)A facets and side (111)A facets. To simulate the growth on the one-sided mesa step, three kinds of wide-enough mesas including a $\langle-110\rangle$-oriented mesa on a $(001)$ substrate, a $\langle-110\rangle$ oriented mesa on a (113)A substrate, and a $\langle-1-12\rangle$ oriented mesa on (111)B substrate, were prepared.

Figures 3(a)-3(c) show the cross-sectional SEM images of the samples after the repeated growth of $\operatorname{AlGaAs}(100 \mathrm{~nm}) /$ $\operatorname{GaAs}(10 \mathrm{~nm})$ layers on one-sided mesa steps formed on (001), (113)A, and (111)B substrates, respectively. In these samples, GaAs layers were used as markers. As seen in Figs. $3(\mathrm{a})-3(\mathrm{c})$, growth behavior on the substrate step is quite different among three kinds of substrates.

In the case of the growth on (001) patterned substrate, a new (113)A facet appeared at the boundary of (111)A and (001) facets of the initial step structure, and it quickly developed during the growth as seen in Fig. 3(a). The likely mechanism of this evolution is schematically shown in Fig. 3(d). Namely, we assume that the growth rate on the (113)A facet is much slower than that on the (001) facet of the initial mesa, but slightly larger than that on the (111)A facet. We also assume that growth rate on high index facets having intermediate facet angles are much larger due to presence of high density steps. Then, at the initial stage, growth on the round portion at the boundary between the top (001) facet and the side (111)A facet of the initial pattern quickly reveals the (113)A facet as shown at the top of Fig. 3(d), because the growth rate has a local minimum at the point where the tangent of growing surface agrees with that of the (113)A facet. Surrounding points have much higher growth rates and grow more quickly so that the round portion quickly evolves into a (113)A facet. After appearance of the (113)A facet, it further develops as schematically shown at the bottom of Fig. 3(d), 


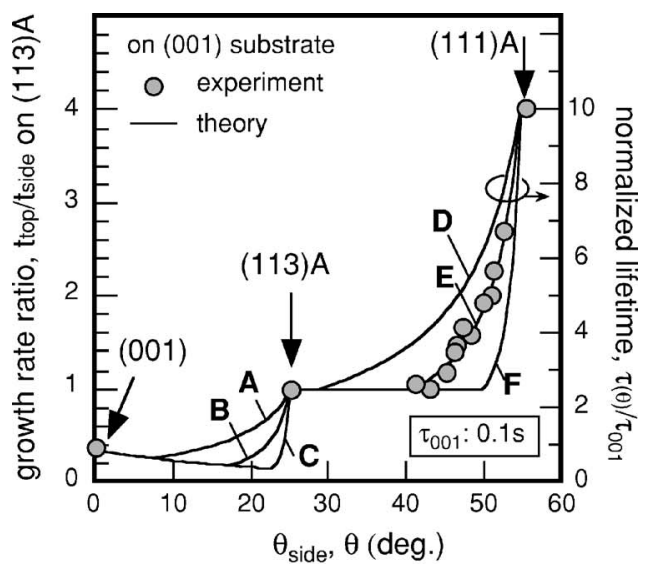

(a)

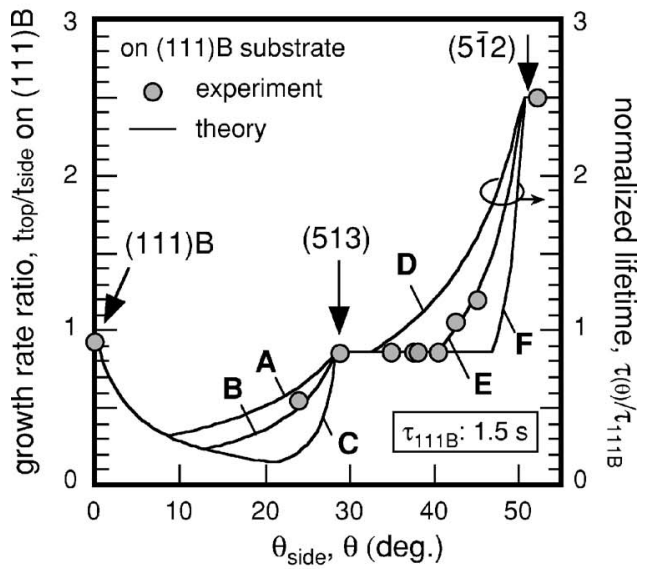

(b)

FIG. 4. Growth rate ratio, $t_{\text {top }} / t_{\text {side }}$ vs $\theta$ determined from Fig. 3 for (a) $\langle-110\rangle$-oriented wires and (b) $\langle-1-12\rangle$-oriented wires. The solid curves indicate the $\theta$ dependence of $\tau(\theta)$ calculated using Eqs. (4a) and (4b) and parameter values listed in Table I.

reflecting the growth rate difference among (001), (113)A, and (111)A facets. Here, $t_{001}, t_{113 \mathrm{~A}}$, and $t_{111 \mathrm{~A}}$ show the vertically grown thicknesses on each of the facets.

Another interesting point in Fig. 3(a) is that the region grown on the top (001) plane is not flat, but nonplanar and shows increased growth rates near the boundary with its exponential decay toward right. This is obviously caused by an additional flux of adatoms from the neighboring side facet by diffusion.

As for the samples grown on the (113)A and (111)B substrates shown in Figs. 3(b) and 3(c), the top of the mesa remained almost planar and flat during repeated growth in both cases, being different from the growth on the (001) substrate. It seems to indicate that the surface diffusion length of adatoms on these planes is much longer as compared with that on the (001) plane. On the other hand, it was found by closely observing Figs. 3(b) and 3(c) that the angle of the side facet of the mesa $\theta_{\text {side }}$ decreases with growth time, as indicated by the dashed lines. This indicates that the material accumulation takes at the bottom corner of the initial mesa step, and it causes a continuous change of the side facet angle $\theta_{\text {side }}$.
The values of the growth rate ratio $t_{\text {top }} / t_{\text {side }}$ measured on $\langle-110\rangle$-oriented mesa step and $\langle-1-12\rangle$-oriented mesa step are plotted in Figs. 4(a) and 4(b), respectively, as a function of side facet angle $\theta_{\text {side }}$. As for the $\langle-110\rangle$-oriented mesa, the data was obtained from the sample grown on the (113)A patterned substrate shown in Fig. 3(b), because it was difficult to define the grown thickness of the sample grown on the (001) patterned substrate due to the previously mentioned variation of growth thickness near the boundary. Here, it should be noted in Fig. 4(a) that the side facet angles on the horizontal axis are those with respect to a (001) plane which were converted from the measured values with respect to the (113)A plane. As for the $\langle-1-12\rangle$-oriented mesa, the growth rate ratio was obtained on the samples grown on (111)B patterned substrates, and the side facet angle $\theta_{\text {side }}$ was measured with respect to the (111)B plane. The solid curves and the right vertical axis shown in Figs. 4(a) and 4(b) are explained later.

As shown in Figs. 4(a) and 4(b), a high growth selectivity on the top planes were obtained at the side facet angle of $\theta_{\text {side }}=55^{\circ}$ for the $\langle-110\rangle$-oriented mesa and $\theta_{\text {side }}=51^{\circ}$ for the $\langle-1-12\rangle$-oriented mesa. These values correspond to the (111)A facet and to the (5-12) facet, respectively. Then, the values of $t_{\text {top }} / t_{\text {side }}$ decreased, as the value of $\theta_{\text {side }}$ decreased. This relation gives important information on the kinetic growth process on various facets.

\section{COMPUTER SIMULATION OF SELECTIVE GROWTH}

\section{A. Basic equations}

The complex behavior of the evolution of cross-sectional structures observed in the experiment is difficult to deal with by a simple analytical treatment. Therefore, an attempt was made here to reproduce the growth profile on the mesa structure by computer simulation based on a phenomenological continuun model. Here, the growth process is described by a diffusion equation with macroscopic parameters such as diffusion constant and lifetime.

We have already carried out such a calculation previously for the growth of the $\langle-1-10\rangle$-oriented QWR on the (001) substrate. ${ }^{19}$ In this modeling, the surface density of group III adatoms $n\left(x, t_{g}\right)$ at the lateral position $x$ and the growth time $t_{g}$ is assumed to satisfy the following phenomenological equation:

$$
\begin{aligned}
& \frac{d n\left(x, t_{g}\right)}{d t_{g}}=G \cos \theta-\frac{n\left(x, t_{g}\right)}{\tau(\theta)}-\frac{d J\left(x, t_{g}\right)}{d x}, \\
& J\left(x, t_{g}\right)=-D \frac{n\left(x, t_{g}\right)}{k_{B} T} \operatorname{grad}(U),
\end{aligned}
$$

where $n\left(x, t_{g}\right)$ is the adatom density, $G$ is the vertically incoming molecular beam flux intensity, and $J$ is the surface diffusion flux intensity of adatoms. $\theta$ is the slope angle defined as the angle with respect to the substrate plane of the tangent of the growing surface taken at the point $x . \tau(\theta)$ is the lifetime of the adatom until it is incorporated into the 
crystal, and it is assumed to be a function of $\theta . D$ and $U$ are the surface diffusion coefficient and the chemical potential of adatoms on a facet, respectively. It should be noted that the above equation is used not only for the planar growing surfaces, but also for the nonplanar growing surfaces. When the surface becomes planar, $\theta$ becomes the side facet angle $\theta_{\text {side }}$ mentioned previously.

After the calculation of the adatom density $n\left(x, t_{g}\right)$ as a function of growth time $t_{g}$, the cross-sectional growth profile is obtained by plotting the vertical growth thickness $T\left(x, t_{g}\right)$, which is represented by the following equation, as a function of lateral position $x$ and growth time $t_{g}$ :

$$
T\left(x, t_{g}\right)=\int_{0}^{t g} \frac{n(x, t) \square \Omega}{\tau \cos \theta} d t,
$$

where $\Omega$ is the volume of the adatom. When there are more than one species of group III adatoms as in the case of growth of AlGaAs, Eq. (2) was solved separately for each species, and each contribution was added together in calculating Eq. (3).

In order to perform the calculation on a computer, values of the various parameters were required. It is obvious that the $\theta$-dependent surface lifetime $\tau(\theta)$ and the diffusion coefficient $D$ of group III adatoms are two very important parameters which determine the evolution of growing surfaces.

\section{B. Lifetime and diffusion constant}

The lifetime of group III adatoms is expected to be more strongly dependent on the step density on the growing surface than on the difference in adatom species. Thus, it is assumed here for simplicity that the surface lifetimes of $\mathrm{Ga}$ adatoms and $\mathrm{Al}$ adatoms are the same. On the other hand, the step density strongly depends on the surface slope $\theta$ and thus the $\theta$ dependence of $\tau(\theta)$ cannot be ignored. In fact, it determines the growth selectivity between the neighboring facets. In our previous work, ${ }^{19}$ we employed a piecewise linear form for $\tau(\theta)$ as a first approximation. In this treatment, $\tau(\theta)$ had local maxima at particular surface slope angles which correspond to (001), (113)A, and (111)A singular facets that appeared in the growth of a $\langle-110\rangle$-oriented wire on a (001) substrate, and these maxima and assumed minima were connected by straight line pieces. Although such a treatment could reproduce the growth evolution rather well by suitable selection of minima, it is rather arbitrary, and does not provide microscopic physical insights for growth

Therefore, we attempted in this study to determine the slope angle dependence of the adatom lifetime by fitting the following formulas by Ohtsuka and Suzuki ${ }^{14,15}$ to the experimentally obtained side facet angle dependence of the growth rate ratio shown in Figs. 4(a) and 4(b):

$$
\begin{aligned}
& \tau(\theta)=\left[1-\alpha_{\text {step }}(\theta)\right] \tau\left(\theta_{\text {sing }}\right), \\
& \alpha_{\text {step }}(\theta)=\frac{L_{0} / \delta_{ \pm}}{1+\left(L_{0} / \delta_{ \pm}\right) \operatorname{coth}\left[\lambda(\theta) / 2 L_{0}\right]} \frac{2 L_{0}}{\lambda(\theta)},
\end{aligned}
$$

$$
\lambda(\theta)=d / \tan \left|\theta-\theta_{\text {sing }}\right|,
$$

where $\alpha_{\text {step }}(\theta)$ is the probability of incorporation of adatoms at the step edge on the growing surface with a slope angle $\theta$. $\tau\left(\theta_{\text {sing }}\right)$ is the lifetime of adatoms on the singular facet with a slope angle of $\theta_{\text {sing }}$ which appears during the selective growth process. $L_{0}$ is the diffusion length of adatoms on the singular facet defined as $L_{0}=\left[\tau\left(\theta_{\text {sing }}\right) D\right]^{1 / 2}$, using the diffusion constant $D . \lambda(\theta)$ is the width of the terrace of the stepterrace structure on the growing surface with a slope angle $\theta$ deviating from $\theta_{\text {sing, }}$, and $d$ is the step height. $\delta_{ \pm}$is a parameter related to the sticking coefficient at the step edge, ${ }^{15}$ and the subscripts \pm correspond to the upward $\left(\theta>\theta_{\text {sing }}\right)$ and downward steps $\left(\theta<\theta_{\text {sing }}\right)$, respectively.

Equations (4a) and (4b) have been obtained by solving a diffusion equation analytically on the terrace with a suitable boundary condition at the step edge. The physics behind these equations is simply the following. As the slope angle deviates from $\theta_{\text {sing }}$, steps appear on the growing surface, and their density increases with increase of $\left|\theta-\theta_{\text {sing }}\right|$. This then enhances the incorporation rate at step edges, and effectively reduces the lifetime.

As for the diffusion constant, we assumed that $D$ has a following strong Arrhenius-type dependence on the substrate temperature $T_{\text {sub }}$ during gowth:

$$
D=D_{0} \exp \left(-E_{d} / k T_{\text {sub }}\right),
$$

where $E_{d}$ is the activation energy of surface diffusion of adatoms.

In our previous papers, ${ }^{19,22}$ the values of $D$ were determined from the repeated growth experiments at various growth temperatures and the quantitative fitting of their growth profiles. Based on these results, the values of $D_{0}$ $=4.0 \times 10^{11} \mathrm{~m}^{2} / \mathrm{s}$ and $E_{d}=4.3 \mathrm{eV}$ were used for the growth on the (001) substrate, ${ }^{19}$ and $D_{0}=1.6 \times 10^{-6} \mathrm{~m}^{2} / \mathrm{s}$ and $E_{d}$ $=1.2 \mathrm{eV}$ were used for the growth on the (111)B substrate, ${ }^{22}$ respectively, in the following simulation.

The present $E_{d}$ value of $4.3 \mathrm{eV}$ obtained for the growth on the (001) substrate is much larger than the value of $0.7 \mathrm{eV}$ reported by Hata et al. ${ }^{23}$ and $2.8 \mathrm{eV}$ reported by Ohta et al., ${ }^{24}$ but it is similar to the value of $4.0 \mathrm{eV}$ reported by Van Hove and Cohen. ${ }^{25}$ The difference in the value of $E_{d}$ seems to be due to the difference in the arsenic pressure used during growth. This is because the diffusion length of adatoms becomes larger under lower arsenic pressure, as reported by Shen et l. $^{26}$ The V/III flux ratio used in this study was 30, and this was much larger than the V/III ratio of 1 used in the experiment by Ohta et al. ${ }^{23}$ On the other hand, Van Hove and Cohen $^{25}$ used a V/III ratio of 10 . Thus, the present $E_{d}$ of 4.3 $\mathrm{eV}$ seems to represent a value under a high arsenic pressure condition.

In the calculations carried out in this study, we assumed that the value of $D$ remains the same on any facets that appeared during growth on the mesa pattern. Furthermore, in order to take account of the migration length difference in $\mathrm{Ga}$ and $\mathrm{Al}$ atoms, we assumed that the value of $D$ for $\mathrm{Al}$ atoms is 100 times smaller that that for $\mathrm{Ga}$ atoms, i.e., $L_{0}(\mathrm{Ga})$ $=10 L_{0}(\mathrm{Al})$, in the calculation. 
TABLE I. Parameters used in the growth simulation.

\begin{tabular}{|c|c|c|c|c|c|c|c|}
\hline $\begin{array}{l}\text { Wire } \\
\text { orientation }\end{array}$ & Facet & $\theta$ [deg.] & $\delta_{+}[\mathrm{m}]$ & $\delta_{-}[\mathrm{m}]$ & $\tau\left(\theta_{\text {sing }}\right)[\mathrm{s}]$ & $D_{0}\left[\mathrm{~m}^{2} / \mathrm{s}\right]$ & $E_{d}[\mathrm{eV}]$ \\
\hline \multirow{7}{*}{$\begin{array}{c}\langle\overline{1} 10\rangle \text { on }(001) \\
\text { substrate }\end{array}$} & (001) & 0 & $5.0 \times 10^{-4}$ & - & 0.1 & $4.0 \times 10^{11}$ & 4.3 \\
\hline & (113)A & 25.2 & $\infty$ & A: $3.0 \times 10^{-4}$ & 0.25 & & \\
\hline & & & & B: $1.0 \times 10^{-4}$ & & & \\
\hline & & & & C: $2.0 \times 10^{-5}$ & & & \\
\hline & (111)A & 54.7 & $\infty$ & D: $3.0 \times 10^{-3}$ & 1.0 & & \\
\hline & & & & E: $1.5 \times 10^{-3}$ & & & \\
\hline & & & & F: $5.0 \times 10^{-4}$ & & & \\
\hline \multirow{7}{*}{$\begin{array}{l}\langle\overline{1} 12\rangle \text { on }(111) \mathrm{B} \\
\quad \text { substrate }\end{array}$} & (111)B & 0 & $7.0 \times 10^{-4}$ & - & 1.5 & $1.6 \times 10^{-6}$ & 1.2 \\
\hline & $(513)$ & 28.6 & $\infty$ & A: $8.0 \times 10^{-4}$ & 1.35 & & \\
\hline & & & & B: $3.0 \times 10^{-4}$ & & & \\
\hline & & & & C: $1.0 \times 10^{-4}$ & & & \\
\hline & $(5 \overline{1} 2)$ & 50.8 & $\infty$ & D: $2.5 \times 10^{-3}$ & 3.75 & & \\
\hline & & & & E: $1.4 \times 10^{-3}$ & & & \\
\hline & & & & F: $5.0 \times 10^{-4}$ & & & \\
\hline
\end{tabular}

Finally, the dependences of lifetime calculated using Eqs. (4a) and (4b) are shown by the solid curves in Fig. 4(a) and 4(b) in terms of the lifetime ratio given on the right vertical axis. Here, the theory and experiments correlated each other by assuming that the measured growth rate ratio corresponds to the lifetime ratio. For the values of the lifetime on the singular facets, we used the data obtained in our previous study. ${ }^{19,22}$ Namely, for growth on the $\langle-110\rangle$ - oriented mesa pattern on the (001) substrate, lifetimes on the (001), (113)A, and (111)A facets denoted as $\tau_{001}, \tau_{113 \mathrm{~A}}$, and $\tau_{111 \mathrm{~A}}$, respectively, were set to satisfy the relation of $\tau_{001}: \tau_{113 \mathrm{~A}}: \tau_{111 \mathrm{~A}}$ $=1: 2.5: 10$ with $\tau_{001}=0.1 \mathrm{~s}$. As for the growth on the $\langle-1-12\rangle$-oriented mesa pattern on the (111)B substrate, lifetimes on (111)B, (513), and (5-12) facets denoted as $\tau_{111 \mathrm{~B}}$, $\tau_{513}$, and $\tau_{5-12}$, respectively, were set as $\tau_{111 \mathrm{~B}}: \tau_{512}: \tau_{5-13}$ $=1: 0.9: 2.5$ with $\tau_{111 \mathrm{~B}}=1.5 \mathrm{~s}$. The diffusion length was calculated from $L_{0}=\left[\tau\left(\theta_{\text {sing }}\right) D\right]^{1 / 2}$, using the above-mentioned values of lifetime and diffusion constant. For calculation of $\lambda(\theta)$, we assumed the presence of monolayer steps on the growing surface. The values of $\delta_{ \pm}$were used as the fitting parameters.

The values of parameters used to generate solid curves A-F in Figs. 4(a) and 4(b) are summarized in Table I. These parameter values were also used for actual calculation of growth profiles explained in the next section. It is seen in Figs. 4(a) and 4(b) that calculated curves can be fitted very well to the measured growth rate ratio. Best fitting was obtained on the combination of curves $\mathrm{B}$ and $\mathrm{E}$ in both cases of growth.

\section{REPRODUCTION OF GROWTH EXPERIMENTS BY SIMULATION}

\section{A. Growth profiles on one-sided mesa step}

In order to see how well the present growth simulation can reproduce the experimental results quantitatively, the growth profiles on one-sided mesa were calculated first for different slope angle dependences of the lifetime $\tau(\theta)$ shown by the solid curves A-F in Figs. 4(a) and 4(b). Figures 5(a) and 5(b) show the calculated growth profiles of $\mathrm{AlGaAs}$ layer with a thickness of $500 \mathrm{~nm}$ on (001) and (111)B patterned substrates, respectively, together with the experimentally observed profiles shown at the bottom. The dashed lines indicate the difference of growth profiles as obtained by subtracting the experimental profile from the calculated one. It is seen in Figs. 5(a) and 5(b) that simulation can reproduce the overall behavior of experimental profile surprisingly well. By more detailed comparison, it is seen that change of the $\tau(\theta)$ curve shown in Figs. 4(a) and 4(b) changes the calculated profiles especially around the region of facet boundaries. The calculation using the $\tau(\theta)$ curves with steep change, such as the curve combination of C-E or B-F, resulted in the growth thickness averaged around facet boundaries. On the other hand, use of $\tau(\theta)$ curves with moderate
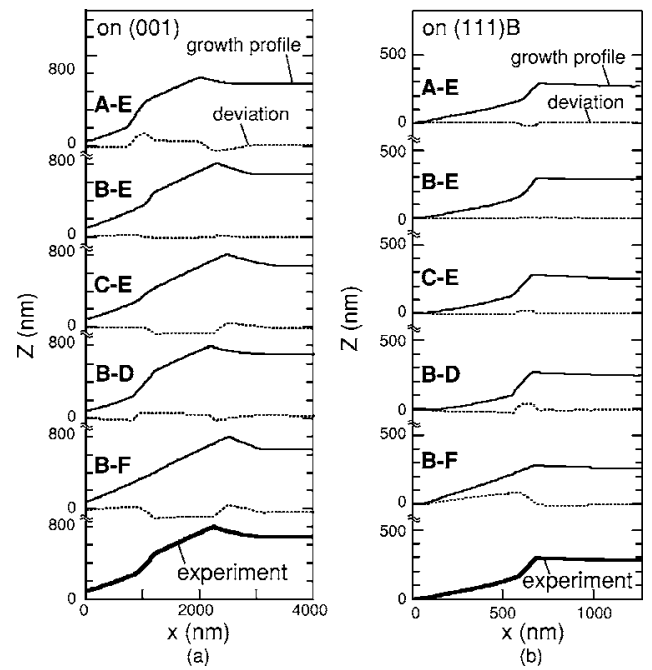

FIG. 5. Calculated growth profiles of AlGaAs layer with $500 \mathrm{~nm}$ grown on (a) $\langle-110\rangle$-oriented mesa on a (001) substrate and (b) $\langle-1-12\rangle$-oriented mesa on a (111)B substrate. The dashed lines indicate the difference between the calculation and the experiment. 


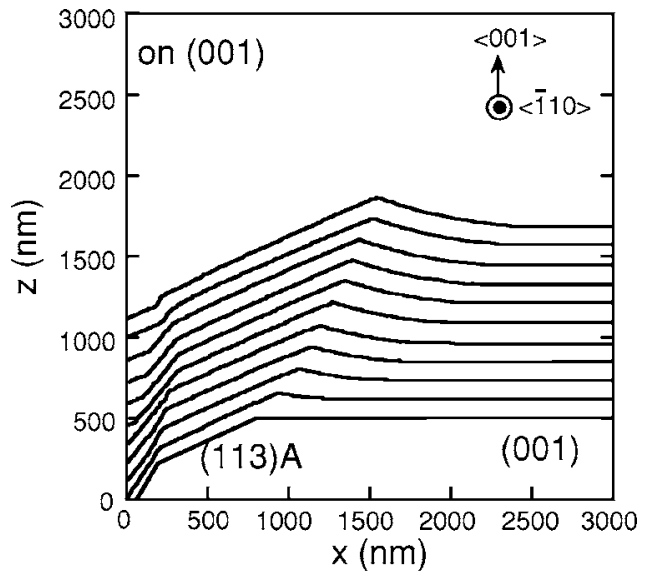

(a)

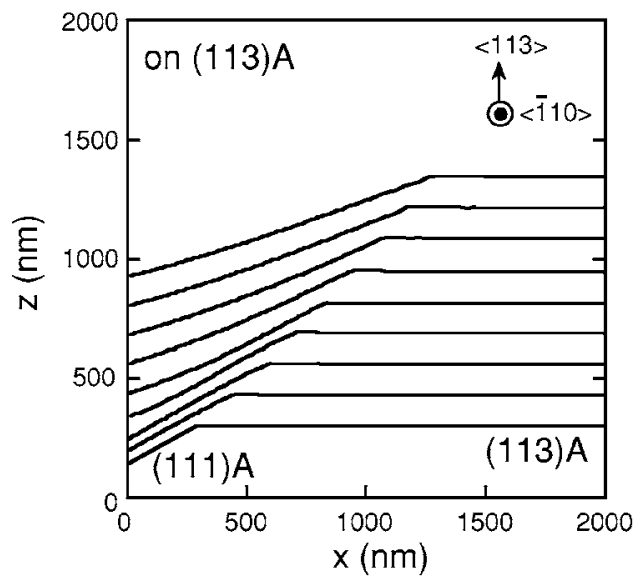

(b)

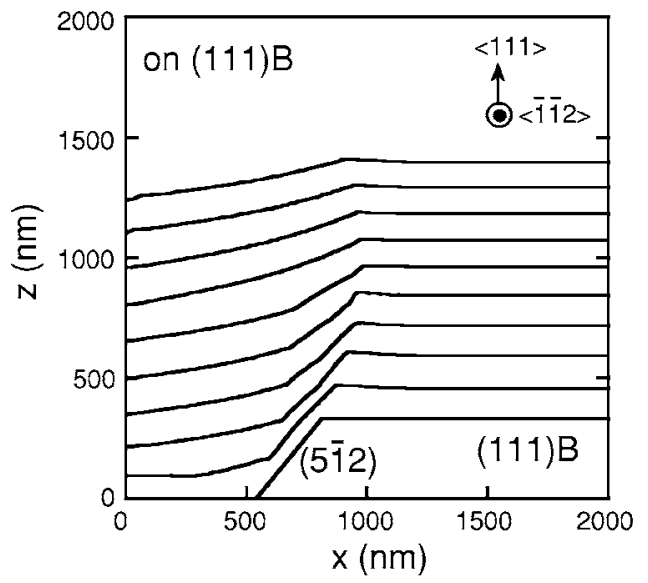

(c)

FIG. 6. Calculated growth profiles of AlGaAs layers for (a) $\langle-110\rangle$-oriented mesa on a (001) substrate, (b) $\langle-110\rangle$-oriented mesa on a (113)A substrate, and $(\mathrm{c})\langle-1-12\rangle$-oriented mesa on a (111)B substrate.

change, such as the curve combination of A-E or B-D, resulted in the large difference of the growth thickness between the neighboring facets. As shown in Figs. 5(a) and 5(b), the curve combination of B-E, which gave best fitting to the measured growth rate ratio as shown in Figs. 4(a) and 4(b), gave the best result in reproducing the experimental growth profiles in both cases.

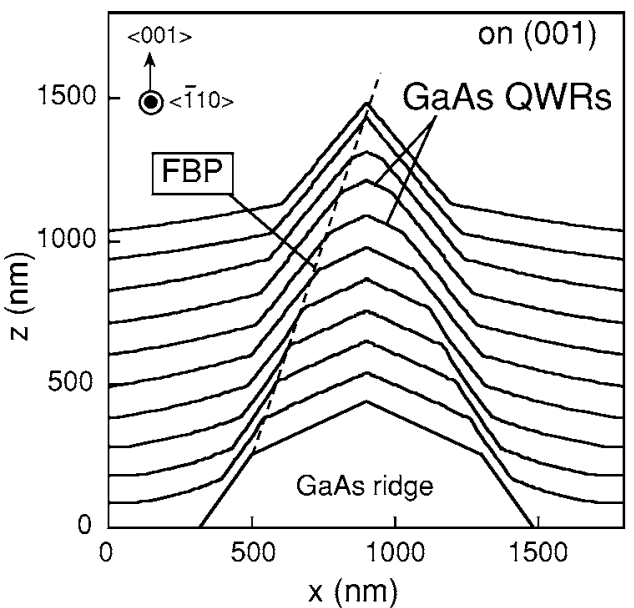

(a)

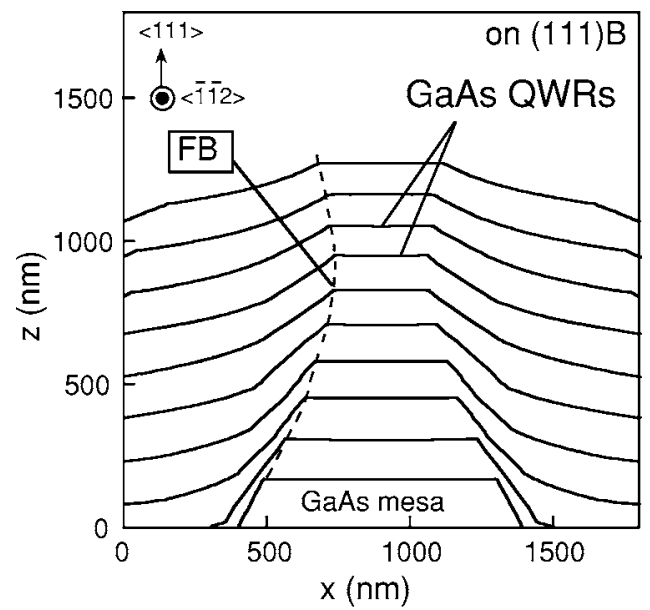

(b)

FIG. 7. Calculated growth profiles of (a) $\langle-1-10\rangle$-oriented GaAs QWR structures on the (001) substrate and (b) $\langle-1-12\rangle$-oriented GaAs QWR structures on the (111)B structures to be compared with experimental ones shown in Figs. 2(a) and 2(b).

The growth profiles calculated on (001), (113)A, and (111)B mesa patterns are shown in Figs. 6(a)-6(c), respectively. As for $\tau(\theta)$, the curve combinations of B-E in Figs. $4(\mathrm{a})$ and $4(\mathrm{~b})$ were used. For the growth on $\langle-110\rangle$-oriented mesa step formed on the (113)A substrate, the same $\tau(\theta)$ curve for growth on the (001) substrate was used. By comparing with the experimentally observed growth profiles shown in Figs. 3(a)-3(c), it is seen that the present growth simulation reproduced very well for all the substrate orientations of the experimentally observed growth profiles including the evolution of facet boundaries.

\section{B. Wire growth and evolution of facet boundary}

Using the above fitting parameters, $\tau(\theta)$ by curves B-E and the diffusion constant $D$, the growth profiles of AlGaAs layers on GaAs ridge or mesa structures were calculated for the actual growth of QWR structures. Figures 7(a) and 7(b) show the theoretical growth profiles calculated for the growth on the $\langle-110\rangle$-oriented mesa on the (001) substrate and for the growth on $\langle-1-12\rangle$-oriented mesa on the (111)B 


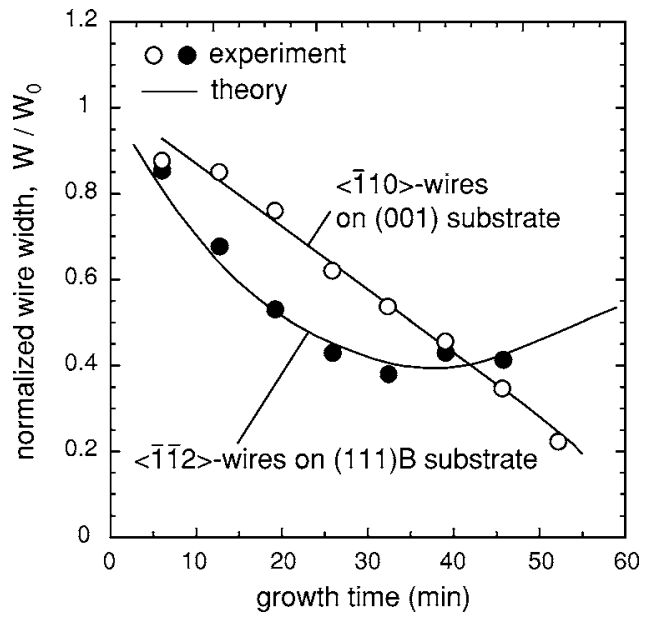

FIG. 8. Experimental and simulated values of the lateral wire width $W$ plotted as functions of the growth time of AlGaAs barrier layers. The data is normalized by the initial width of GaAs buffer ridge or mesa structures $W_{0}$.

substrate, respectively. Growth profiles very similar to experimental ones were obtained. The facet boundaries are clearly seen in the simulation and they determine the lateral width $W$ of QWRs. Thus, formation of facet boundary planes is a consequence of the difference of growth rates between the neighboring facets caused by incorporation and lateral diffusion of adatoms. The values of $W$ obtained theoretically are compared with the experimentally obtained values in Fig. 8. As for the $\langle-110\rangle$-oriented wires, the lateral wire width decreased linearly with the growth time of AlGaAs layers supplied before the GaAs wire formation. On the other hand, the width of $\langle-1-12\rangle$-oriented wires did not change linearly with the growth thickness. This is due to the difference in the growth kinetics which strongly depends on crystalline facets. As shown in Fig. 8, the theoretical width obtained by the present simulation is in very good agreement with those obtained by the experiments. These results indicate that the lateral width of present QWRs can be kinetically controlled by the growth conditions and the supply thickness of the AlGaAs layer prior the start of wire growth. The present simulation seems to be very powerful for precise control of wire shape, width, and height.

\section{CONCLUSION}

In this study, the mechanism of the cross-sectional evolution during the selective MBE growth of $\langle-110\rangle-$ and $\langle-1-12\rangle$-oriented GaAs QWRs was studied both experimentally and theoretically. From the detailed investigation on the growth profiles, it was found that the lateral wire width is determined by facet boundaries within AlGaAs layers. Detailed features of cross-sectional structures including evolution of facet boundaries could be well reproduced for growth on (001), (113)A, and (111)B substrates by a computer simulation based on a phenomenological continuum growth model where the slope angle dependence of lifetime of adatoms was duly taken into account both theoretically and experimentally. It is concluded that the lateral width of present QWRs can be kinetically controlled by the growth conditions and the supply thickness of AlGaAs layers. The present simulation seems to be powerful for such purposes.

\section{ACKNOWLEDGMENTS}

The work reported here is supported in part by the $21 \mathrm{st}$ Century COE Project at Hokkaido University on "MemeMedia Technology Approach to the R\&D of Next-Generation Information Technologies" and by a Grant-in-Aid for Young Scientists (B)-16760239 both from the Japanese Government.

${ }^{1}$ E. Kapon, D. M. Hwang, and R. Bhat, Phys. Rev. Lett. 63, 430 (1989). ${ }^{2}$ T. Fukui, S. Ando, Y. Tokura, and T. Toriyama, Appl. Phys. Lett. 58, 2018 (1991).

${ }^{3}$ S. Koshiba, H. Noge, H. Akiyama, T. Inoshita, Y. Nakamura, A. Shimizu, Y. Nagamune, M. Tsuchiya, H. Kano, H. Sakaki, and K. Wada, Appl. Phys. Lett. 64, 363 (1994).

${ }^{4}$ X. L. Wang, M. Ogura, and H. Matsuhata, Appl. Phys. Lett. 66, 1506 (1995).

${ }^{5}$ H. Fujikura and H. Hasegawa, J. Electron. Mater. 25, 619 (1996).

${ }^{6}$ T. Muranaka, C. Jiang, A. Ito, and H. Hasegawa, Thin Solid Films 380 , 189 (2000)

${ }^{7}$ T. Kimura, T. Fukushi, T. Muranaka, T. Sato, and H. Hasegawa, International Conference on Indium Phosphide and Related Materials (IPRM '04), Kagoshima, Japan, 2004 (The Japan Society of Applied Physics, IEEE Lasers and Electro-Optics Society, IEEE Electron Devices Society, 2004).

${ }^{8}$ T. Sato, I. Tamai, C. Jiang, and H. Hasegawa, Inst. Phys. Conf. Ser. 170-4, 325 (2002)

${ }^{9}$ T. Sato, I. Tamai, and H. Hasegawa, Inst. Phys. Conf. Ser. 174-3, 145 (2003).

${ }^{10}$ I. Tamai, S. Yoshida, T. Sato, and H. Hasegawa, Physica E (Amsterdam) 21, 521 (2004)

${ }^{11}$ T. Takebe, M. Fujii, T. Yamamoto, K. Fujita, and T. Watanabe, J. Appl. Phys. 81, 7273 (1997).

${ }^{12}$ N. Haider, M. R. Wilby, and D. D. Vvedensky, J. Cryst. Growth 127, 922 (1993).

${ }^{13}$ K. Asano, Y. Kangawa, H. Ishizaki, T. Akiyama, K. Nakamura, and T. Ito, Appl. Surf. Sci. 237, 206 (2004).

${ }^{14}$ M. Ohtsuka and A. Suzuki, J. Appl. Phys. 73, 7358 (1993).

${ }^{15}$ M. Ohtsuka, J. Cryst. Growth 205, 112 (1999).

${ }^{16}$ M. Ozdemir and A. Zangwill, J. Vac. Sci. Technol. A 10, 684 (1992).

${ }^{17}$ P. S. Dominguez and F. Briones, Microelectron. J. 26, 751 (1995).

${ }^{18}$ G. Biasiol, A. Gustafsson, K. Leifier, and E. Kapon, Phys. Rev. B 65 , 205306 (2002).

${ }^{19}$ T. Sato, I. Tamai, and H. Hasegawa, J. Vac. Sci. Technol. B 22, 2266 (2004).

${ }^{20}$ S. Yoshida, I. Tamai, T. Sato, and H. Hasegawa, Jpn. J. Appl. Phys., Part 1 43, 2064 (2004).

${ }^{21}$ T. Sato, I. Tamai, S. Yoshida, and H. Hasegawa, Appl. Surf. Sci. 234, 11 (2004).

${ }^{22}$ I. Tamai, T. Sato, and H. Hasegawa, Jpn. J. Appl. Phys., Part 1 44, 2652 (2005).

${ }^{23}$ M. Hata, T. Isu, A. Watanabe, and Y. Katayama, J. Vac. Sci. Technol. B 8, $692(1990)$.

${ }^{24}$ K. Ohta, T. Kojima, and T. Nakagawa, J. Cryst. Growth 95, 71 (1989).

${ }^{25}$ J. M. Van Hove and P. I. Cohen, J. Cryst. Growth 81, 13 (1987).

${ }^{26}$ X. Q. Shen, D. Kishimoto, and T. Nishinaga, Jpn. J. Appl. Phys., Part 1 33, 11 (1994). 